\title{
Gordon and Kerr-Schild ansätze in massive and bimetric gravity
}

\author{
Valentina Baccetti, Prado Martin-Moruno, and Matt Visser \\ School of Mathematics, Statistics, and Operations Research, \\ Victoria University of Wellington, PO Box 600, Wellington 6140, New Zealand \\ E-mail: valentina.baccetti@msor.vuw.ac.nz; prado@msor.vuw.ac.nz; \\ matt.visser@msor.vuw.ac.nz
}

AbStRACt: We develop the "generalized Gordon ansatz" for the ghost-free versions of both massive and bimetric gravity, an ansatz which is general enough to include almost all spacetimes commonly considered to be physically interesting, and restricted enough to greatly simplify calculations. The ansatz allows explicit calculation of the matrix square root $\gamma=\sqrt{g^{-1} f}$ appearing as a central feature of the ghost-free analysis. In particular, this ansatz automatically allows us to write the effective stress-energy tensor as that corresponding to a perfect fluid. A qualitatively similar "generalized KerrSchild ansatz" can also be easily considered, now leading to an effective stress-energy tensor that corresponds to a null fluid. Cosmological implications are considered, as are consequences for black hole physics. Finally we have a few words to say concerning the null energy condition in the framework provided by these ansätze.

Keywords: graviton mass, massive gravity, bimetric gravity, background geometry, foreground geometry, Gordon metric, Kerr-Schild metric.

Thursday 21 June 2012; HTEX-ed May 21, 2018. 


\section{Contents}

1 Introduction 2

2 Stress energy tensor in bimetric gravity 2

3 Generalized Gordon ansatz $\quad 5$

3.1 Historical background 6

$\begin{array}{lll}3.2 & \text { Perfect fluid effective stress-energy tensor } & 7\end{array}$

4 Generality of the ansatz $\quad 9$

4.1 Cosmological solutions 9

4.2 Massive-gravity black holes $\quad 12$

4.2.1 Isotropic coordinates 13

4.2.2 Horizon-penetrating coordinates 13

5 Generalized Kerr-Schild ansatz $\quad 15$

$\begin{array}{lll}6 & \text { Discussion } & 17\end{array}$ 


\section{Introduction}

The ghost-free massive and bimetric gravities have recently attracted considerable attention. Early (pre-ghost-free) work included [1-3], while notable contributions to the recent ghost-free discussion include [4-12]. Recent work on the relation between massive gravity and bimetric gravity has been reported in [13], and the status of the null energy condition (NEC) is explored in [14]. Cosmological implications are discussed in [15-21], while the status of black holes is considered in [22-29].

An overarching theme of all the ghost-free technical calculations is the need for

manipulating and working with the matrix square root $\gamma=\sqrt{g^{-1} f}$ built out of the foreground and background metrics. Some particularly useful mathematical results related to dealing with this quantity are reported in [14]. In the current article we shall investigate a specific ansatz for the relation between foreground and background metrics that is sufficiently general to be able to represent almost all spacetimes of physical interest, while being simultaneously sufficiently special to make computations simple. The ansatz is inspired by the "analogue spacetime" programme [30-32], but has wide applicability and validity outside of that original context. (Note that the analogue spacetime programme could play a relevant role in this context not only as an inspiration, but even as a scenario where bimetric models could naturally emerge [33].)

The paper is organized as follows: The general framework for ghost-free massive and bimetric gravity is very briefly summarized in section 2 . Then in section 3 we introduce a particularly powerful ansatz which can be obtained by generalizing the Gordon metric (normally arising in relativistic optics). This "generalized Gordon ansatz" allows us to write the effective stress-energy tensor as that of a perfect fluid, see subsection 3.2. Despite the rather strong conclusions implied by this ansatz, it is still general enough to describe the most common physically interesting spacetimes considered in the literature - see in particular subsection 4. In section 5 we present the "generalized Kerr-Schild ansatz" and discuss the implied stress-energy tensor, now that of a null fluid. We conclude with a discussion in section 6 .

\section{Stress energy tensor in bimetric gravity}

The action of bimetric gravity can be expressed quite generally as $[13,14]$

$$
\begin{aligned}
S= & -\frac{1}{16 \pi G} \int d^{4} x \sqrt{-g}\left\{R(g)+2 \Lambda-2 m^{2} L_{\text {int }}(g, f)\right\}+S_{(\mathrm{m})} \\
& -\frac{\kappa}{16 \pi G} \int d^{4} x \sqrt{-f}\{\bar{R}(f)+2 \bar{\Lambda}\}+\epsilon \bar{S}_{(\mathrm{m})} .
\end{aligned}
$$


Here $S_{(\mathrm{m})}$ and $\bar{S}_{(\mathrm{m})}$ are the usual matter actions, with the foreground and background matter fields being coupled only to the foreground and background metrics $g_{\mu \nu}$ and $f_{\mu \nu}$, respectively. All interactions between these two sectors are confined to the term $L_{\text {int }}(g, f)$, which is an algebraic function of $g$ and $f$. We can recover the action of massive gravity by considering $\kappa=\epsilon=0$ [13]. In this case, we would have an aether theory in which the dynamics of the physical metric $g_{\mu \nu}$ depends on a now non-dynamical background metric $f_{\mu \nu}$. (Up to this point, the analysis is identical to the pre-ghost-free analyses of the 1970s [1, 2], and later [3].)

As is now well-known [4], the action (2.1) is ghost-free if and only if the interaction term can be written as a linear combination of the elementary symmetric polynomials of the eigenvalues of the matrix $\gamma=\sqrt{g^{-1} f}$. In view of the results of [14] we can write

$$
f_{\mu \nu}=\gamma_{\mu}^{\sigma} g_{\sigma \rho} \gamma_{\nu}^{\rho} ; \quad \text { that is } \quad f=\gamma^{T} g \gamma .
$$

We can then, in 3+1 dimensions, express the interaction Lagrangian as [13]

$$
L_{\mathrm{int}}=\alpha_{1} e_{1}(\gamma)+\alpha_{2} e_{2}(\gamma)+\alpha_{3} e_{3}(\gamma)
$$

where as usual

$$
\begin{aligned}
& e_{1}(\gamma)=\operatorname{tr}[\gamma] \\
& e_{2}(\gamma)=\frac{1}{2}\left(\operatorname{tr}[\gamma]^{2}-\operatorname{tr}\left[\gamma^{2}\right]\right) \\
& e_{3}(\gamma)=\frac{1}{6}\left(\operatorname{tr}[\gamma]^{3}-3 \operatorname{tr}[\gamma] \operatorname{tr}\left[\gamma^{2}\right]+2 \operatorname{tr}\left[\gamma^{3}\right]\right)
\end{aligned}
$$

The two remaining non-vanishing polynomials, $e_{0}(\gamma)=1$ and $e_{4}(\gamma)=\operatorname{det}(\gamma)$, have been absorbed into the foreground and background cosmological constants. The three parameters appearing in the interaction term are not fully independent. If one additionally requires that $L_{\text {int }}$ should (for weak fields) be of the canonical Fierz-Pauli form, then $[7,13]$

$$
\alpha_{1}+2 \alpha_{2}+\alpha_{3}=-1
$$

Both metrics have exactly the same status. The interaction term satisfies the reciprocity relation $[4,13]$

$$
\sqrt{-g} L_{\mathrm{int}}(\gamma)=\sqrt{-g} \sum_{i=0}^{4} \alpha_{i} e_{i}(\gamma)=\sqrt{-f} \sum_{i=0}^{4} \alpha_{4-i} e_{i}\left(\gamma^{-1}\right)=\sqrt{-f} \bar{L}_{\mathrm{int}}\left(\gamma^{-1}\right)
$$

That is, the entire theory could be equivalently re-expressed using $f$ as the foreground metric and $g$ as the background. Varying the action (2.1) with respect the two metrics, we obtain two sets of equations of motion [13]:

$$
G^{\mu}{ }_{\nu}-\Lambda \delta^{\mu}{ }_{\nu}=m^{2} T^{\mu}{ }_{\nu}+8 \pi G T_{\nu}^{(\mathrm{m}) \mu}
$$


and

$$
\kappa\left(\bar{G}_{\nu}^{\mu}-\bar{\Lambda} \delta_{\nu}^{\mu}\right)=m^{2} \bar{T}_{\nu}^{\mu}+\epsilon 8 \pi G \bar{T}_{\nu}^{(\mathrm{m}) \mu}
$$

Here

$$
T^{\mu}{ }_{\nu}=\tau_{\nu}^{\mu}-\delta^{\mu}{ }_{\nu} L_{\mathrm{int}}
$$

and

$$
\bar{T}_{\nu}^{\mu}=-\frac{\sqrt{-g}}{\sqrt{-f}} \tau_{\nu}^{\mu}
$$

with

$$
\tau_{\nu}^{\mu}=\gamma_{\rho}^{\mu} \frac{\partial L_{\text {int }}}{\partial \gamma_{\rho}^{\nu}}
$$

The indices of equation (2.9) and (2.10) must be raised and lowered using $g$ and $f$, respectively. The equations of motion of the $g$-space ( $f$-space) are modified with respect to those of general relativity by the introduction of an effective stress-energy tensor associated to the interaction between the two geometries (and the quantity $\kappa / \epsilon$ ). It is a slightly non-trivial exercise, see [14], to verify that the effective stress-energy tensors are indeed symmetric. In massive gravity with a non-dynamical background one would only have the first set of equation of motions (2.9), see [13, 19]. Both effective stressenergy tensors fulfill the Bianchi-inspired constraints

$$
\nabla_{\mu} T_{\nu}^{\mu}=0 ; \quad \bar{\nabla}_{\mu} \bar{T}_{\nu}^{\mu}=0
$$

Once one constraint is enforced, for example $\nabla_{\mu} T^{\mu}{ }_{\nu}=0$, then the other is also automatically fulfilled [19, 20].

The modifications to the two sets of equations of motion are very closely related. Everything can be expressed in terms of a single mixed-index tensor $\tau^{\mu}{ }_{\nu}$, which can be written as a cubic polynomial in the matrix $\gamma$. Specifically [14]

$$
\tau_{\nu}^{\mu}=\left(\alpha_{1}+\alpha_{2} e_{1}(\gamma)+\alpha_{3} e_{2}(\gamma)\right) \gamma_{\nu}^{\mu}-\left(\alpha_{2}+\alpha_{3} e_{1}(\gamma)\right)\left\{\gamma^{2}\right\}^{\mu}{ }_{\nu}+\alpha_{3}\left\{\gamma^{3}\right\}^{\mu}{ }_{\nu}
$$

This yields the effective stress-energy tensor as viewed by the $g$-space, up to a cosmological constant contribution coming from second term in the RHS of equations (2.11). Explicitly

$$
T_{\nu}^{\mu}=-\left(\alpha_{1} e_{1}(\gamma)+\alpha_{2} e_{2}(\gamma)+\alpha_{3} e_{3}(\gamma)\right) \delta_{\nu}^{\mu}+\tau_{\nu}^{\mu}
$$

We also note the foreground-background symmetry

$$
\sqrt{-g} T_{\nu}^{\mu}+\sqrt{-f} \bar{T}_{\nu}^{\mu}=-\sqrt{-g} L_{\text {int }}(\gamma) \delta^{\mu}{ }_{\nu}=-\sqrt{-f} \bar{L}_{\text {int }}\left(\gamma^{-1}\right) \delta^{\mu}{ }_{\nu}
$$




\section{Generalized Gordon ansatz}

Let us now consider the following ansatz relating the two metrics:

$$
f_{\mu \nu}=\Omega^{2}\left\{g_{\mu \nu}+\xi V_{\mu} V_{\nu}\right\}
$$

Here $V^{\mu}$ is a unit timelike vector with respect to $g_{\mu \nu}$, so that $g_{\mu \nu} V^{\mu} V^{\nu}=-1$, and we set $V_{\mu}=g_{\mu \nu} V^{\nu}$. Furthermore $\Omega$ and $\xi$ are arbitrary functions. Noting that both metrics must have Lorentzian signature, we see $\xi<1$. So we can write $\xi=1-\zeta^{2}$, with $\zeta \neq 0$. Furthermore, without any loss of generality we can choose $\zeta$ to be strictly positive, that is $\zeta>0$. Then

$$
f_{\mu \nu}=\Omega^{2}\left\{\left(g_{\mu \nu}+V_{\mu} V_{\nu}\right)-\zeta^{2} V_{\mu} V_{\nu}\right\}
$$

Mathematically, this ansatz can be thought of a conformal transformation $\Omega$, combined with a stretch $\zeta$ along the timelike direction parallel to $V$. Note that (see figure 1 ) the

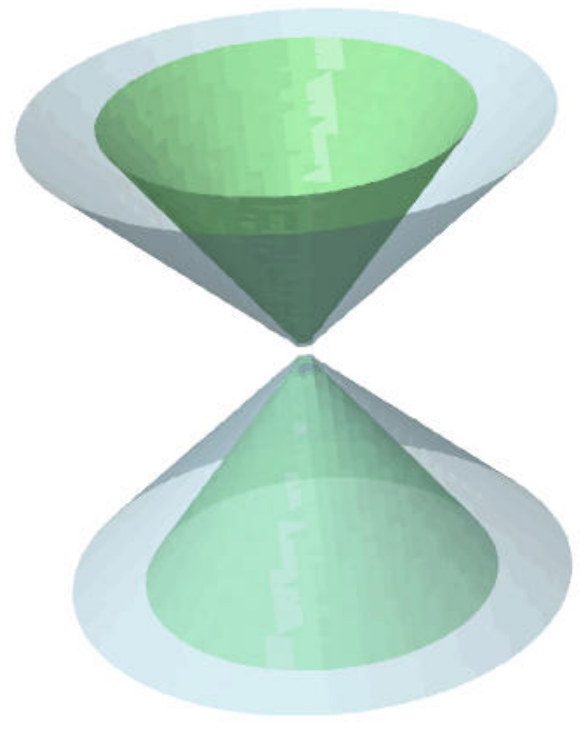

Figure 1. Light cones for the generalized Gordon ansatz: Depending on the value of the parameter $\zeta$ the foreground light cones lie strictly inside, on top of, or strictly outside the background light cones.

relative position of the light cones is very strongly correlated. 
- For $0<\zeta<1$ the light cones of $f$ lie strictly inside the light cones of $g$.

- For $\zeta=1$ the light cones of $f$ and $g$ coincide; the two metrics are conformally related.

- For $\zeta>1$ the light cones of $f$ lie strictly outside the light cones of $g$.

\subsection{Historical background}

Physically, this ansatz is a significant generalization of Gordon's 1923 metric, which was originally developed within the context of geometric optics [34]; see particularly [30, 31] for recent discussions. The original Gordon metric, which describes ray optics in an optical medium of 4 -velocity $V^{\mu}$ and constant refractive index $n$ in Minkowski space, is expressed as

$$
\mathcal{G}_{\mu \nu}=\left(\eta_{\mu \nu}+V_{\mu} V_{\nu}\right)-\frac{1}{n^{2}} V_{\mu} V_{\nu}
$$

where $V_{\mu}=\eta_{\mu \nu} V^{\nu}$. Our ansatz (3.2) corresponds to a generalization of (3.3). One should:

(i) Generalize the refractive index $n$ to be a position-dependent function $\left(1 / n^{2} \rightarrow \zeta^{2}\right)$;

(ii) Introduce a position-dependent conformal factor $\Omega$, (which does not affect the light cone structure);

(iii) Consider a non-trivial input metric $\left(g_{\mu \nu}\right.$ instead of $\left.\eta_{\mu \nu}\right)$;

(iv) Relabel the output metric $\left(f_{\mu \nu}\right.$ instead of $\left.\mathcal{G}_{\mu \nu}\right)$.

For this reason, we call our ansatz the "generalized Gordon ansatz". The ansatz can also naturally be inverted to express $g_{\mu \nu}$ in terms of $f_{\mu \nu}$ :

$$
g_{\mu \nu}=\Omega^{-2}\left\{\left(f_{\mu \nu}+\bar{V}_{\mu} \bar{V}_{\nu}\right)-\zeta^{-2} \bar{V}_{\mu} \bar{V}_{\nu}\right\},
$$

where $\bar{V}$ is now normalized in terms of $f_{\mu \nu} \bar{V}^{\mu} \bar{V}^{\nu}=-1$, and we set $\bar{V}_{\mu}=f_{\mu \nu} \bar{V}^{\nu}$. For consistency we must then enforce $\bar{V}_{\mu}=\Omega \zeta V_{\mu}$ and $\bar{V}^{\mu}=\Omega^{-1} \zeta^{-1} V^{\mu}$.

This generalized Gordon ansatz also has exactly the same structure as the (geometric) relativistic acoustic metric of [35-37]; see particularly [30, 31] for recent discussions. Our ansatz, as expressed in equation (3.2), exactly agrees with the form of the geometric acoustic metric appearing in reference [35-37]. (Here we have denoted the second metric $f_{\mu \nu}$ instead of $\mathcal{G}_{\mu \nu}$. Furthermore in the present situation $\zeta^{2}$ is not necessarily restricted to be less than one. In contrast, in [35, 37] $\zeta^{2}=c_{s}^{2} / c^{2} \leq 1$, simply because the speed of sound is subluminal, though "tachyacoustic cosmologies", based on [36], are now fashionable.) 


\subsection{Perfect fluid effective stress-energy tensor}

Using this ansatz we can express the matrix $\gamma$ in a simple form by noting that

$$
g^{\mu \sigma} f_{\sigma \nu}=\Omega^{2}\left\{\left(\delta^{\mu}{ }_{\nu}+V^{\mu} V_{\nu}\right)-\zeta^{2} V^{\mu} V_{\nu}\right\}=\left(\gamma^{2}\right)^{\nu}{ }_{\nu} .
$$

Then the matrix square root is easy to extract

$$
\gamma^{\mu}{ }_{\nu}=\left(\sqrt{g^{-1} f}\right)^{\mu}{ }_{\nu}=\Omega\left\{\left(\delta^{\mu}{ }_{\nu}+V^{\mu} V_{\nu}\right)-\zeta V^{\mu} V_{\nu}\right\} .
$$

Furthermore

$$
\left(\gamma^{n}\right)^{\mu}{ }_{\nu}=\Omega^{n}\left\{\left(\delta^{\mu}{ }_{\nu}+V^{\mu} V_{\nu}\right)-\zeta^{n} V^{\mu} V_{\nu}\right\} .
$$

The symmetric polynomials appearing in the interaction term (2.3), given by equations (2.4), also take on a particularly simple form. They are

$$
e_{1}(\gamma)=\Omega\{3+\zeta\} ; \quad e_{2}(\gamma)=3 \Omega^{2}\{1+\zeta\} ; \quad e_{3}(\gamma)=\Omega^{3}\{1+3 \zeta\} .
$$

Note that these expressions are linear in $\zeta$. This can be traced back to the fact that only one of the eigenvalues of $\gamma$ is at all $\zeta$-dependent, and that in a linear fashion. Taking into account expressions (3.5), (3.6), and (3.8), we see that equations (2.15) and (2.16), imply that the stress-energy tensor takes a perfect fluid form:

$$
T^{\mu}{ }_{\nu}=p\left(\delta^{\mu}{ }_{\nu}+V^{\mu} V_{\nu}\right)+\rho V^{\mu} V_{\nu} .
$$

Here we have defined

$$
\rho=\Omega\left(3 \alpha_{1}+3 \alpha_{2} \Omega+\alpha_{3} \Omega^{2}\right)
$$

and

$$
p=-\Omega\left[\left(2 \alpha_{1}+\alpha_{2} \Omega\right)+\left(\alpha_{1}+2 \alpha_{2} \Omega+\alpha_{3} \Omega^{2}\right) \zeta\right] .
$$

Note that these expressions are also linear in $\zeta$, ultimately for the same reason as above. It is also useful to write

$$
\tau_{\nu}^{\mu}=\hat{p}\left(\delta^{\mu}{ }_{\nu}+V^{\mu} V_{\nu}\right)+\hat{\rho} V^{\mu} V_{\nu},
$$

defining

$$
\hat{\rho}=-\Omega \zeta\left(\alpha_{1}+3 \alpha_{2} \Omega+3 \alpha_{3} \Omega^{2}\right),
$$

and

$$
\hat{p}=\Omega\left[\alpha_{1}+\alpha_{2} \Omega(2+\zeta)+\alpha_{3} \Omega^{2}(1+2 \zeta)\right] .
$$

Here we have used the fact that $T^{\mu}{ }_{\nu}$ and $\tau^{\mu}{ }_{\nu}$ only differ in a cosmological constant contribution. Moreover, we have

$$
\rho+p=\hat{\rho}+\hat{p}=\Omega\left(\alpha_{1}+2 \alpha_{2} \Omega+\alpha_{3} \Omega^{2}\right)(1-\zeta) .
$$


Note that $\zeta=1(\xi=0)$ corresponds to a cosmological constant, as should be expected, since in this case ansatz (3.1) reduces to a conformal relation between the two metrics.

Moreover, due to the Bianchi-inspired constraint (2.14), the stress-energy tensor not only takes the perfect fluid form (3.9), but also obeys the usual conservation equations. These are

$$
V^{\mu} \nabla_{\mu} \rho+(\rho+p) \nabla^{\mu} V_{\mu}=0
$$

and

$$
(\rho+p) V^{\mu} \nabla_{\mu} V_{\nu}+\left(g_{\mu \nu}+V_{\mu} V_{\nu}\right) \nabla^{\mu} p=0 .
$$

On the other hand, in view of equations (3.10) and (3.15), it can be noted that the effective fluid automatically fulfills the additional constraint:

$$
\nabla_{\mu} \rho=3 \frac{\rho+p}{1-\zeta} \frac{\nabla_{\mu} \Omega}{\Omega}
$$

From equations (3.16) and (3.18) one can conclude that, once $\Omega$ and $V^{a}$ have been fixed in (3.2), the following equation is automatically fulfilled

$$
\zeta=\frac{\nabla_{a}\left(\Omega^{3} V^{\mu}\right)}{\Omega^{3} \nabla_{\mu} V^{\mu}} .
$$

Finally, the effective stress-energy tensor in the $f$-space (2.12) also takes on a perfect fluid form

$$
\bar{T}_{\nu}^{\mu}=\bar{p}\left(\delta_{\nu}^{\mu}+\bar{V}^{\mu} \bar{V}_{\nu}\right)+\bar{\rho} \bar{V}^{\mu} \bar{V}_{\nu} .
$$

Now $\bar{V}_{\mu}=\Omega \zeta V_{\mu}$, as defined in the previous section, is normalized in terms of $f_{\mu \nu} \bar{V}^{\mu} \bar{V}^{\nu}=-1$, and so

$$
\bar{p}=-\frac{1}{\Omega^{4} \zeta} \hat{p} ; \quad \bar{\rho}=-\frac{1}{\Omega^{4} \zeta} \hat{\rho} .
$$

(This last step could equivalently be obtained by noting $\sqrt{-f}=\Omega^{4} \zeta \sqrt{-g}$.) Perhaps more tellingly

$$
\bar{\rho}+\bar{p}=-\frac{1}{\Omega^{4} \zeta}(\hat{\rho}+\hat{p})=-\frac{1}{\Omega^{4} \zeta}(\rho+p) .
$$

Thus, the sum of the effective energy density plus the pressure of the effective perfect fluid due to the modification of general relativity in the background space has opposite sign to that characterizing the effective perfect fluid in the foreground space. (This is a special case of the more general NEC-violating behaviour discussed in [14]. For general background on the NEC and its variants, see [38-41].) Some of the possible consequences of this sign flip are discussed in the particular case of cosmological solutions in section 4. 


\section{Generality of the ansatz}

Up to now, we have shown that the ansatz expressed through (3.1) or, equivalently, through (3.2), implies that the bimetric-induced effective stress-energy tensor takes the form of a perfect fluid. Moreover, the ansatz constrains the effects of the modifications of the theory in the two spaces so tightly that (apart from the "trivial" case of pure cosmological constant) the violation of the NEC condition in one space follows straightforwardly from the fulfillment of the NEC in the other space [14]. Nevertheless, the ansatz is general enough to describe many different physically relevant situations. Some particularly relevant examples are considered below.

\subsection{Cosmological solutions}

Let us now assume that the metric $g_{\mu \nu}$ takes the form of a FLRW spacetime in a certain coordinate patch. This is

$$
d s_{g}^{2}=-d t^{2}+a(t)^{2}\left[\frac{d r^{2}}{1-k r^{2}}+r^{2}\left\{d \theta^{2}+\sin ^{2} \theta d \phi^{2}\right\}\right] .
$$

Application of the ansatz (3.2) leads to

$$
d s_{f}^{2}=-\Omega(t)^{2}\left\{\zeta(t)^{2} d t^{2}+a(t)^{2}\left[\frac{d r^{2}}{1-k r^{2}}+r^{2}\left\{d \theta^{2}+\sin ^{2} \theta d \phi^{2}\right\}\right]\right\} .
$$

This metric can be written as

$$
d s_{f}^{2}=-N(t)^{2} d t^{2}+b(t)^{2}\left[\frac{d r^{2}}{1-k r^{2}}+r^{2}\left\{d \theta^{2}+\sin ^{2} \theta d \phi^{2}\right\}\right],
$$

where the scale factor and lapse function of metric $f$ are

$$
b(t)=\Omega(t) a(t)
$$

and

$$
N(t)=\Omega(t) \zeta(t)
$$

respectively. Thus, the assumption of the generalized Gordon ansatz (3.2), when considering a cosmological foreground metric, implies a cosmological background metric with a different scale factor and cosmic time, as should be expected. The only restrictions coming from forcing that relation is that the two metrics should both be diagonal in the same coordinate patch, and have the same sign of spatial curvature $k$, conditions that certainly should be expected to be fulfilled by general cosmological solutions $[20,21]$. 
The conservation equations (3.16) take on the usual cosmological form. Thus

$$
\dot{\rho}+3(\rho+p) \frac{\dot{a}}{a}=0 .
$$

Taking into account equation (3.15) and the derivative of equation (3.10), this implies

$$
\frac{\dot{\Omega}}{\Omega}=-(1-\zeta) \frac{\dot{a}}{a} .
$$

So, defining the Hubble parameters for both metrics in the usual way, $H_{g}=\dot{a} / a$ and $H_{f}=\dot{b} / b$, and taking into account the definition of $b(4.4)$, we see that the two Hubble parameters are very tightly intertwined

$$
H_{f}=\zeta H_{g} .
$$

This is equivalent to the relation obtained in reference [21] to characterize their "branch two" solutions, the general class of cosmological solutions in bimetric gravity.

Moreover, rewriting (4.7) as

$$
\frac{\dot{\Omega}}{\Omega}+\frac{\dot{a}}{a}=\zeta \frac{\dot{a}}{a},
$$

in view of (4.4) we see

$$
\frac{\dot{b}}{b}=\zeta \frac{\dot{a}}{a},
$$

whence

$$
\zeta=\frac{\dot{b}}{\dot{a}} \frac{a}{b}=\frac{d \ln b / d t}{d \ln a / d t} .
$$

Taking into account (4.5) and (4.4), this implies a lapse function

$$
N(t)=\frac{\dot{b}}{\dot{a}} .
$$

So, in this case, one can write the metric of the background space (4.3) using only one arbitrary function $b(t)$ :

$$
d s_{f}^{2}=-\left(\frac{\dot{b}}{\dot{a}}\right)^{2} d t^{2}+b(t)^{2}\left[\frac{d r^{2}}{1-k r^{2}}+r^{2}\left\{d \theta^{2}+\sin ^{2} \theta d \phi^{2}\right\}\right] .
$$

Moreover, from equations (4.4) and (4.11), we can express the energy density (3.10) and pressure (3.11) of the effective stress energy tensor in the foreground space as

$$
\rho=\frac{b}{a}\left(3 \alpha_{1}+3 \alpha_{2} \frac{b}{a}+\alpha_{3} \frac{b^{2}}{a^{2}}\right),
$$


and

$$
p=-\frac{b}{a}\left(2 \alpha_{1}+\alpha_{2} \frac{b}{a}\right)-\left(\alpha_{1}+2 \alpha_{2} \frac{b}{a}+\alpha_{3} \frac{b^{2}}{a^{2}}\right) \frac{\dot{b}}{\dot{a}},
$$

respectively. The $g$-space Bianchi-inspired conservation equation is then automatically satisfied for arbitrary $b(t)$.

Let us briefly consider the implications on the cosmological evolution of the $g$ space and $f$-space due to the inter-twining relation between the NECs. The simplest possibility is to have both NECs satisfied, which in this cosmological setting implies that the two cosmologies are conformally related, with the contribution to the effective stress-energy being an arbitrary cosmological constant that can be tuned at will. (It must be emphasized that, in principle, more general solutions are possible for particular combinations of the parameters appearing in $L_{\text {int }}$.)

Nevertheless, the case when the NECs are not saturated is more interesting, because either the foreground or background geometry has a NEC-violating effective stressenergy tensor. Assuming that the pathologies are relegated to the "other" gravitational sector, that is, in our own sector $\rho>0$ and $p+\rho>0$, then, in view of equations (3.10), (3.13), (3.15) and (3.21), we have two possibilities:

(i) Either $\bar{\rho}>0$ and $\bar{p}+\bar{\rho}<0$, which is phantom energy.

(ii) Or $\bar{\rho}<0$ and $\bar{p}+\bar{\rho}<0$, which corresponds to an even weirder kind of cosmological fluid known as dual dark energy [42].

Regarding (i), as is well known, phantom energy can easily lead to a big rip [43], big freeze [44, 45], future singularities [46], and other oddities [47-50]. The only possibility to avoid future doomsday in this scenario, under the assumption $p+\rho>0$, seems to be by requiring $\bar{p}+\bar{\rho} \rightarrow 0$ asymptotically (implying the same behaviour for $p+\rho$ ), leading to a cosmology which approximates a de Sitter behaviour at late times. In the second case, (ii), it must be noted that this effective dual dark energy is compatible with a Lorentzian geometry $\left(3 H_{f}^{2}=m^{2} \bar{\rho} / \kappa+\bar{\Lambda}-k / b^{2}>0\right)$, a positive cosmological constant $\bar{\Lambda}>0$ (with $\bar{\Lambda}=\Lambda_{f}+\alpha_{4}$ ), or in a spatially hyperbolic universe, $k<0$. As was studied in reference [51] for the case of a constant ratio $\bar{w}=\bar{p} / \bar{\rho}$, a universe filled with dual dark energy and equipped with a positive cosmological constant (with $k=0$ ) is completely regular and the $\bar{\Lambda}$-term dominates at late times. Thus, in this case, the $f$-universe is asymptotically de Sitter at late times (for $\bar{\Lambda}>0$ and any $k$ ).

On the other hand, in massive gravity we are free to choose any arbitrary function $b(t)$ since there are no equations of motion (2.10) for the background metric. Therefore, we can use the graviton mass terms to generate by hand an effective stress-energy contributing to cosmological fluid that has any properties we desire - the large scale 
evolution of our universe is no longer governed by the matter in our universe, but can instead be forced to dance to the tune determined by the arbitrarily specified background geometry (see reference [19], and for earlier [pre-ghost-free] speculations along these lines see [3]).

\subsection{Massive-gravity black holes}

As we have already pointed out, the present ansatz can be motivated by generalizing the Gordon metric (and the relativistic acoustic metric) appearing in the field of analogue spacetimes [30, 31]. Following this spirit, and in view of the achievements of that field, one might expect this ansatz to be able to describe black hole geometries, at least when the metric describing the "medium" is the Minkowski metric. It must be noted that considering one of the metrics to be flat would implicitly restrict our attention to massive gravity, where one of the metrics is non-dynamical. This is the theory that we are implicitly assuming in the present subsection.

Furthermore, let us note that a Minkowski medium in expression (3.3) implies $g_{\mu \nu}=\eta_{\mu \nu}$, so $g_{\mu \nu}$ cannot be the dynamical metric, whereas it is $\mathcal{G}_{\mu \nu}=f_{\mu \nu}$ that is dynamical. Usually in massive gravity the physical dynamical metric is denoted by $g_{\mu \nu}$ and the background metric is $f_{\mu \nu}$, but this is simply a matter of notation. (Recall the symmetry of the interaction Lagrangian (2.8) which leads to the graviton contribution to the matter Lagrangian in massive gravity.) We maintain the notation of (3.3) for consistency with the analogue model programme. (In particular, all the results of this subsection would remain unchanged by adopting and specializing the "inverse ansatz" (3.4), considering $g_{\mu \nu}$ to be the dynamical metric and $f_{\mu \nu}=\eta_{\mu \nu}$ to be non-dynamical, and introducing an extra normalization factor in the four-velocity (4.19); since, in that case, it would be $V$ that was a timelike unit vector with respect to the curved metric, while $\bar{V}$ would be a timelike unit vector with respect to the Minkowski metric.)

It should be pointed out that assuming a flat background for massive gravity is not too strong an assumption when dealing with black holes, (though it would certainly be an overly stringent assumption for massive-gravity cosmology), at least as long as we are not presuming that both metrics can be written in a diagonal form in the same coordinate patch [28]. (See also [29].) In fact, to our knowledge, massive-gravity black holes (not bimetric gravity black holes) have been studied in the literature only by assuming flat backgrounds [22-25].

In massive gravity with a conformally flat background metric, our ansatz (3.1) can be written as

$$
f_{\mu \nu}=\Omega^{2}\left\{\eta_{\mu \nu}+\xi V_{\mu} V_{\nu}\right\}
$$

There are at least two ways of proceeding. 


\subsubsection{Isotropic coordinates}

Take $V^{\mu}=(1, \mathbf{0})$, and set $U^{2}=\Omega^{2}(1-\xi)$. Then

$$
d s^{2}=-U^{2} d t^{2}+\Omega^{2}\|d \mathbf{x}\|^{2}
$$

This puts the metric into isotropic coordinates - and it is well known that this form of the metric is certainly sufficient (in principle) to deal with the Schwarzschild and Reissner-Nordström geometries, though the Kerr and Kerr-Newman geometries cannot be put in this form [52]. However in this coordinate system $\gamma=\sqrt{g^{-1} f}$ is singular (in the matrix sense) as any horizon (corresponding to $U=0$ ) is approached. Thus once $L_{\text {int }}$ is "turned on" diagonal coordinate systems of this type behave unpleasantly the stress tensor $T^{\mu}{ }_{\nu}$ is then a singular matrix at the horizon, and other approaches might be more profitable.

\subsubsection{Horizon-penetrating coordinates}

Let us now adopt horizon-penetrating coordinates. Choose $\Omega=1$, and express the background metric in the following gauge:

$$
d s_{\eta}^{2}=\eta_{\mu \nu} d x^{\mu} d x^{\nu}=-d t^{2}+d r^{2}+r^{2}\left\{d \theta^{2}+\sin ^{2} \theta d \phi^{2}\right\}
$$

Take the four-velocity to be

$$
V^{\mu}=\gamma\left(1,-\beta^{i}\right) ; \quad V_{\mu}=-\gamma\left(1, \beta^{i}\right) ; \quad \beta^{i}=\left(\beta^{r}, 0,0\right)
$$

Note that the usual relation between $\beta$ and $\gamma$ is recovered when the normalization of $V_{\mu}$ with respect to $\eta_{\mu \nu}$ is imposed. (The $\gamma$ appearing here should not be confused with the matrix $\gamma=\sqrt{g^{-1} f}$ depending on the two metrics; its form would be that of the usual relativistic quantity. The intended meaning should be clear from context.) Thus, taking into account (4.16), (4.18) and (4.19), we can write

$$
d s_{f}^{2}=-\left(1-\xi \gamma^{2}\right) d t^{2}+2 \xi \gamma \sqrt{\gamma^{2}-1} d t d r+\left[1+\xi\left(\gamma^{2}-1\right)\right] d r^{2}+r^{2} d \Omega_{(2)}^{2}
$$

Here $d \Omega_{(2)}^{2}=d \theta^{2}+\sin ^{2} \theta d \phi^{2}$ must not be confused with the conformal factor $\Omega$.

Let us now consider the specific function

$$
\gamma(r)=\sqrt{1+K / r}
$$

Then the foreground metric (4.20) can be written as

$$
d s_{f}^{2}=-\left(1-\xi-\frac{\xi K}{r}\right) d t^{2}+2 \xi \frac{\sqrt{K(K+r)}}{r} d t d r+\left(1+\frac{\xi K}{r}\right) d r^{2}+r^{2} d \Omega_{(2)}^{2}
$$


In its current form it is not canonically normalized at spatial infinity. If we now re-define the time variable as $\tilde{t}=\sqrt{1-\xi} t$, then

$$
d s_{f}^{2}=-\left(1-\frac{\xi K}{(1-\xi) r}\right) d \tilde{t}^{2}+2 \xi \frac{\sqrt{K(K+r)}}{\sqrt{1-\xi} r} d \tilde{t} d r+\left(1+\frac{\xi K}{r}\right) d r^{2}+r^{2} d \Omega_{(2)}^{2}
$$

which by explicit computation can easily be seen to be Ricci flat. This allows us to identify

$$
2 m=\frac{\xi K}{(1-\xi)}
$$

and so

$d s_{f}^{2}=-\left(1-\frac{2 m}{r}\right) d \tilde{t}^{2}+2 \frac{\sqrt{2 m[2 m(1-\xi)+\xi r]}}{r} d \tilde{t} d r+\left(1+\frac{2 m(1-\xi)}{r}\right) d r^{2}+r^{2} d \Omega_{(2)}^{2}$.

Now clearly in the limit $\xi \rightarrow 1$ one recovers the Painlevé-Gullstrand form of the Schwarzschild metric, whereas if $\xi=0$, then the black hole is expressed using the Eddington-Finkelstein metric. (For a related analysis, see [53].)

One might think that there is some problem in performing a time re-definition once the gauge freedom is completely used to fix the form of the background metric (4.18). However, the same result can be obtained in a slightly different manner without explicitly performing any coordinate change. Let us start from the background metric already written using the new time coordinate. This is

$$
d s_{\eta}^{2}=\tilde{\eta}_{\mu \nu} d \tilde{x}^{\mu} d \tilde{x}^{\nu}=-\frac{1}{(1-\xi)} d \tilde{t}^{2}+d r^{2}+r^{2}\left\{d \theta^{2}+\sin ^{2} \theta d \phi^{2}\right\}
$$

Considering now

$$
\tilde{V}^{\mu}=\gamma\left(\sqrt{1-\xi},-\beta^{i}\right) ; \quad \tilde{V}_{\mu}=-\gamma\left(\frac{1}{\sqrt{1-\xi}}, \beta^{i}\right)
$$

with $\gamma$ given by equation (4.21), and

$$
\tilde{f}_{\mu \nu}=\tilde{\eta}_{\mu \nu}+\xi \tilde{V}_{\mu} \tilde{V}_{\nu}
$$

one can recover directly the foreground metric as given in (4.25). In this coordinate system the matrix $\gamma=\sqrt{g^{-1} f}$ and its inverse are non-singular (in the matrix sense) as any horizon is approached. Thus once $L_{\text {int }}$ is "turned on", the stress tensor $T^{\mu}{ }_{\nu}$ is a non-singular matrix at the horizon, and can easily be tuned to be perturbatively small. Turning on the massive graviton should then not qualitatively disturb the horizon structure. 
Thus we see that the generalized Gordon ansatz is sufficiently flexible to deal with Schwarzschild black holes (and Reissner-Nordström black holes). This observation is important in that it gives us confidence that the ansatz is not too restrictive - the physics results we are obtaining above really are due to the interaction Lagrangian, not due to an overly restrictive metric ansatz.

\section{Generalized Kerr-Schild ansatz}

We now consider an ansatz which can be thought as being inspired by the Kerr-Schild metrics $[54,55]$. This ansatz takes a similar form to that considered in the previous section, namely

$$
f_{\mu \nu}=\Omega^{2}\left(g_{\mu \nu}+\xi l_{\mu} l_{\nu}\right)
$$

where the vector $l_{\mu}$ is now a null vector for foreground metric $g_{\mu \nu} l^{\mu} l^{\nu}=0$. So the geometries in question are conformally Kerr-Schild. It should be emphasized that the Kerr-Schild ansatz is an important and rather general one, which includes almost all spacetimes of physical interest $[54,55]$. Note that (see figure 2):

- For $\xi<0$, apart from a single null vector in common, the light cones of $f$ lie strictly outside the light cones of $g$.

- For $\xi=0$ the light cones of $f$ and $g$ coincide; the two metrics are conformally related. (In this particular case the generalized Gordon and generalized KerrSchild ansätze coincide.)

- For $\xi>0$, apart from a single null vector in common, the light cones of $f$ lie strictly inside the light cones of $g$.

For this ansatz, one has

$$
g^{\mu \sigma} f_{\sigma \nu}=\Omega^{2}\left\{\delta_{\nu}^{\mu}+\xi l^{\mu} l_{\nu}\right\}=\left(\gamma^{2}\right)^{\mu}{ }_{\nu}
$$

whence

$$
\gamma_{\nu}^{\mu}=\Omega\left\{\delta^{\mu}{ }_{\nu}+\frac{1}{2} \xi l^{\mu} l_{\nu}\right\}
$$

and in general

$$
\left(\gamma^{n}\right)^{\mu}{ }_{\nu}=\Omega^{n}\left\{\delta^{\mu}{ }_{\nu}+\frac{n}{2} \xi l^{\mu} l_{\nu}\right\}
$$

The symmetric polynomials are given by

$$
e_{1}(\gamma)=4 \Omega ; \quad e_{2}(\gamma)=6 \Omega^{2} ; \quad e_{3}(\gamma)=4 \Omega^{3}
$$




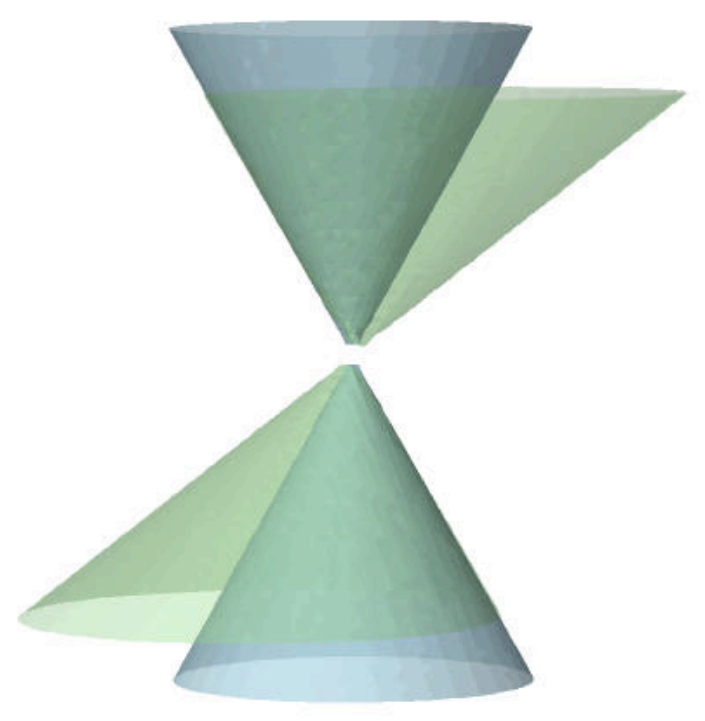

Figure 2. Light cones for the generalized Kerr-Schild ansatz: Depending on the value of the parameter $\xi$, and apart from one null ray in common, the foreground light cones lie strictly inside, on top of, or strictly outside the background light cones.

(Note these quantities are independent of $\xi$.) Thus, taking into account equations (2.15) and (2.16), the stress-energy tensor can in this situation be written as

$$
T^{\mu}{ }_{\nu}=\mathcal{F} l^{\mu} l_{\nu}+\Xi \delta^{\mu}{ }_{\nu}
$$

where

$$
\mathcal{F}=-\frac{\Omega}{2} \xi\left(\alpha_{1}+2 \Omega \alpha_{2}+\Omega^{2} \alpha_{3}\right)
$$

and

$$
\Xi=-\Omega\left(3 \alpha_{1}+3 \Omega \alpha_{2}+\alpha_{3} \Omega^{2}\right)
$$

Note that this stress-energy tensor can be interpreted as a null fluid (with null flux $\mathcal{F}$, and pressure $\Xi$ ). This is a specialization of the "type II" stress-energy tensor considered by Hawking and Ellis [56]. Satisfaction of the NEC in this situation is 
checked by contracting with a generic null vector (not necessarily $l^{\mu}$ ), and amounts to the condition $\mathcal{F} \geq 0$.

We can also write $(2.15)$

$$
\tau_{\nu}^{\mu}=\hat{\mathcal{F}} l^{\mu} l_{\nu}+\hat{\Xi} \delta^{\mu}{ }_{\nu}
$$

By inspection

$$
\hat{\mathcal{F}}=\mathcal{F} ; \quad \hat{\Xi}=\Xi+L_{\text {int }}=\Omega\left(\alpha_{1}+3 \alpha_{2} \Omega+\alpha_{3} \Omega^{2}\right)
$$

Finally,

$$
\bar{T}_{\nu}^{\mu}=\overline{\mathcal{F}} l^{\mu} l_{\nu}+\bar{\Xi} \delta^{\mu}{ }_{\nu} .
$$

Noting that now $\sqrt{-f}=\Omega^{4} \sqrt{-g}$, we have

$$
\overline{\mathcal{F}}=-\frac{1}{\Omega^{4}} \hat{\mathcal{F}} ; \quad \bar{\Xi}=-\frac{1}{\Omega^{4}} \hat{\Xi} .
$$

Regarding the NEC the key observation is

$$
\overline{\mathcal{F}}=-\frac{1}{\Omega^{4}} \mathcal{F} .
$$

We again very explicitly see how foreground and background NECs are anti-correlated [14].

\section{Discussion}

In the technical analysis of ghost-free bimetric gravity a central role is played by the matrix square root $\gamma=\sqrt{g^{-1} f}$ relating the foreground and background metrics. Motivated by the fact that this object can be somewhat awkward to deal with, we have developed two lines of attack. In a companion paper we have developed a number of mathematical results that help us manipulate this quantity [14], ultimately leading to a rather general analysis of the interplay between the foreground and background NECs. In the current article we have taken a slightly different tack, and have characterized the circumstances under which it is possible to simply and explicitly compute this matrix square root.

In order to simplify the problem we have assumed that the metrics are related in a very particular form, fulfilling what we called the "generalized Gordon ansatz". This ansatz can be motivated from the analogue spacetime programme, and has been proven to be amazingly powerful in this paper. It implies that the effective stress-energy tensor automatically takes the form of a perfect fluid. This ansatz is extremely natural in a 
cosmological setting, and helps one understand why bimetric FLRW cosmologies have proved to be relatively tractable.

Moreover, it has also allowed us to recover easily the relation between the NECs in the two spaces [14]. That is, we have shown that the simultaneous fulfillment of the NEC associated to the modifications of general relativity in both foreground and background spaces is only possible if it saturates; that is if the effect of these modifications are equivalent to the consideration of a cosmological constant.

In counterpoint to these rather strong conclusions, it should be noted that the generalized Gordon ansatz seems sufficiently general to study the most common physical situations one might be interested in. In fact, we have shown that it can describe the general class of cosmological solutions in bimetric gravity, as well as black holes in massive gravity.

We have also considered a less general ansatz based on the Kerr-Schild metrics. The effective stress-energy tensor in this case has not quite so natural a form; it is equivalent to a null fluid. Nevertheless, even in this case similar conclusions can be extracted about the simultaneous fulfillment of both NECs - this is only possible in the trivial case in which both metrics have the same light cone structure.

\section{Acknowledgments}

VB acknowledges support by a Victoria University PhD scholarship. PMM acknowledges financial support from the Spanish Ministry of Education through a FECYT

grant, via the postdoctoral mobility contract EX2010-0854. MV acknowledges support via the Marsden Fund and via a James Cook Research Fellowship, both administered by the Royal Society of New Zealand.

\section{References}

[1] C. J. Isham, A. Salam and J. A. Strathdee, "F-dominance of gravity", Phys. Rev. D 3 (1971) 867.

[2] D. G. Boulware and S. Deser, "Can gravitation have a finite range?", Phys. Rev. D 6 (1972) 3368.

[3] M. Visser, "Mass for the graviton", Gen. Rel. Grav. 30 (1998) 1717 [gr-qc/9705051].

[4] S. F. Hassan and R. A. Rosen, "Bimetric Gravity from Ghost-free Massive Gravity", JHEP 1202 (2012) 126 [arXiv:1109.3515 [hep-th]].

[5] C. de Rham and G. Gabadadze, "Generalization of the Fierz-Pauli Action", Phys. Rev. D 82 (2010) 044020 [arXiv:1007.0443 [hep-th]]. 
[6] C. de Rham, G. Gabadadze, and A. J. Tolley, "Resummation of Massive Gravity", Phys. Rev. Lett. 106 (2011) 231101 [arXiv:1011.1232 [hep-th]].

[7] S. F. Hassan and R. A. Rosen, "On Non-Linear Actions for Massive Gravity", JHEP 1107 (2011) 009 [arXiv:1103.6055 [hep-th]].

[8] S. F. Hassan and R. A. Rosen, "Resolving the Ghost Problem in non-Linear Massive Gravity", Phys. Rev. Lett. 108 (2012) 041101 [arXiv:1106.3344 [hep-th]].

[9] S. F. Hassan, R. A. Rosen, and A. Schmidt-May, "Ghost-free Massive Gravity with a General Reference Metric", JHEP 1202 (2012) 026 [arXiv:1109.3230 [hep-th]].

[10] S. F. Hassan and R. A. Rosen, "Confirmation of the Secondary Constraint and Absence of Ghost in Massive Gravity and Bimetric Gravity", JHEP 1204 (2012) 123 [arXiv:1111.2070 [hep-th]].

[11] S. F. Hassan, A. Schmidt-May and M. von Strauss, "Proof of Consistency of Nonlinear Massive Gravity in the Stückelberg Formulation", arXiv:1203.5283 [hep-th].

[12] K. Hinterbichler and R. A. Rosen, "Interacting Spin-2 Fields", arXiv:1203.5783 [hep-th].

[13] V. Baccetti, P. Martin-Moruno and M. Visser, "Massive gravity from bimetric gravity", arXiv:1205.2158 [gr-qc].

[14] V. Baccetti, P. Martin-Moruno and M. Visser, "Null Energy Condition violations in bimetric gravity", arXiv:1206.3814 [gr-qc].

[15] C. de Rham and L. Heisenberg, "Cosmology of the Galileon from Massive Gravity", Phys. Rev. D 84 (2011) 043503 [arXiv:1106.3312 [hep-th]].

[16] A. H. Chamseddine and M. S. Volkov, "Cosmological solutions with massive gravitons", Phys. Lett. B 704 (2011) 652 [arXiv:1107.5504 [hep-th]].

[17] G. D'Amico, C. de Rham, S. Dubovsky, G. Gabadadze, D. Pirtskhalava, and A. J. Tolley, "Massive Cosmologies", Phys. Rev. D 84 (2011) 124046 [arXiv:1108.5231 [hep-th]].

[18] A. E. Gumrukcuoglu, C. Lin and S. Mukohyama, "Open FRW universes and self-acceleration from nonlinear massive gravity", JCAP 1111 (2011) 030 [arXiv:1109.3845 [hep-th]].

[19] M. von Strauss, A. Schmidt-May, J. Enander, E. Mortsell and S. F. Hassan, "Cosmological Solutions in Bimetric Gravity and their Observational Tests", JCAP 1203 (2012) 042 [arXiv:1111.1655 [gr-qc]].

[20] M. S. Volkov, "Cosmological solutions with massive gravitons in the bigravity theory", JHEP 1201 (2012) 035 [arXiv:1110.6153 [hep-th]].

[21] D. Comelli, M. Crisostomi, F. Nesti, and L. Pilo, "FRW Cosmology in Ghost Free 
Massive Gravity from Bigravity", JHEP 1203 (2012) 067 [arXiv:1111.1983 [hep-th]].

[22] K. Koyama, G. Niz and G. Tasinato, "Analytic solutions in non-linear massive gravity", Phys. Rev. Lett. 107 (2011) 131101 [arXiv:1103.4708 [hep-th]].

[23] K. Koyama, G. Niz and G. Tasinato, "Strong interactions and exact solutions in non-linear massive gravity", Phys. Rev. D 84 (2011) 064033 [arXiv:1104.2143 [hep-th]].

[24] L. Berezhiani, G. Chkareuli, C. de Rham, G. Gabadadze and A. J. Tolley, "On Black Holes in Massive Gravity", Phys. Rev. D 85 (2012) 044024 [arXiv:1111.3613 [hep-th]].

[25] F. Sbisa, G. Niz, K. Koyama and G. Tasinato, "Characterising Vainshtein Solutions in Massive Gravity", arXiv:1204.1193 [hep-th].

[26] D. Comelli, M. Crisostomi, F. Nesti and L. Pilo, "Spherically Symmetric Solutions in Ghost-Free Massive Gravity", Phys. Rev. D 85 (2012) 024044 [arXiv:1110.4967 [hep-th]].

[27] M. S. Volkov, "Hairy black holes in the ghost-free bigravity theory", arXiv:1202.6682 [hep-th].

[28] C. Deffayet and T. Jacobson, "On horizon structure of bimetric spacetimes", Class. Quant. Grav. 29 (2012) 065009 [arXiv:1107.4978 [gr-qc]].

[29] M. Bañados, A. Gomberoff and M. Pino, "The bigravity black hole and its thermodynamics", Phys. Rev. D 84 (2011) 104028 [arXiv:1105.1172 [gr-qc]].

[30] M. Visser, "Survey of analogue spacetimes", Submitted to the proceedings of the IX'th SIGRAV graduate school: Analogue Gravity, Lake Como, Italy, May 2011, arXiv:1206.2397 [gr-qc].

[31] C. Barceló, S. Liberati and M. Visser, "Analogue gravity", Living Rev. Rel. 8 (2005) 12 [Living Rev. Rel. 14 (2011) 3] [gr-qc/0505065].

[32] M. Visser, "Acoustic black holes: Horizons, ergospheres, and Hawking radiation", Class. Quant. Grav. 15 (1998) 1767 [gr-qc/9712010].

[33] G. Jannes and G. E. Volovik, "The cosmological constant: A lesson from the effective gravity of topological Weyl media", arXiv:1108.5086 [gr-qc].

[34] W. Gordon, "Zur Lichtfortpflanzung nach der Relativitätstheorie", Ann. Phys. (Leipzig), 72 (1923) 421-456. doi: 10.1002/andp.19233772202

[35] V. Moncrief, "Stability of stationary, spherical accretion onto a Schwarzschild black hole", Astrophys. J., 235 (1980) 1038-1046.

[36] E. Babichev, V. Mukhanov and A. Vikman, "k-Essence, superluminal propagation, causality and emergent geometry", JHEP 0802 (2008) 101 [arXiv:0708.0561 [hep-th]].

[37] M. Visser and C. Molina-París, "Acoustic geometry for general relativistic barotropic irrotational fluid flow", New J. Phys. 12 (2010) 095014 [arXiv:1001.1310 [gr-qc]]. 
[38] C. Barceló and M. Visser, "Twilight for the energy conditions?", Int. J. Mod. Phys. D 11 (2002) 1553 [gr-qc/0205066].

[39] G. Abreu, C. Barceló and M. Visser, "Entropy bounds in terms of the $w$ parameter", JHEP 1112 (2011) 092 [arXiv:1109.2710 [gr-qc]].

[40] L. H. Ford and T. A. Roman, "Averaged energy conditions and quantum inequalities", Phys. Rev. D 51 (1995) 4277 [gr-qc/9410043].

[41] L. H. Ford and T. A. Roman, "The Quantum interest conjecture", Phys. Rev. D 60 (1999) 104018 [gr-qc/9901074].

[42] A. V. Yurov, P. Martín-Moruno and P. F. González-Díaz, "New Bigs in cosmology", Nucl. Phys. B 759 (2006) 320 [astro-ph/0606529].

[43] R. R. Caldwell, M. Kamionkowski and N. N. Weinberg, "Phantom energy and cosmic doomsday", Phys. Rev. Lett. 91 (2003) 071301 [astro-ph/0302506].

[44] M. Bouhmadi-López, P. F. González-Díaz and P. Martín-Moruno, "Worse than a big rip?", Phys. Lett. B 659 (2008) 1 [gr-qc/0612135].

[45] M. Bouhmadi-López, P. F. González-Díaz and P. Martín-Moruno, "On the generalised Chaplygin gas: Worse than a big rip or quieter than a sudden singularity?", Int. J. Mod. Phys. D 17 (2008) 2269 [arXiv:0707.2390 [gr-qc]].

[46] C. Cattoën and M. Visser, "Necessary and sufficient conditions for big bangs, bounces, crunches, rips, sudden singularities, and extremality events", Class. Quant. Grav. 22 (2005) 4913 [gr-qc/0508045].

[47] C. Molina-París and M. Visser, "Minimal conditions for the creation of a Friedman-Robertson-Walker universe from a bounce", Phys. Lett. B 455 (1999) 90 [gr-qc/9810023].

[48] D. Hochberg, C. Molina-París and M. Visser, "Tolman wormholes violate the strong energy condition", Phys. Rev. D 59 (1999) 044011 [gr-qc/9810029].

[49] M. Visser, "Energy conditions in the epoch of galaxy formation", Science 276 (1997) 88.

[50] M. Visser, "General relativistic energy conditions: The Hubble expansion in the epoch of galaxy formation", Phys. Rev. D 56 (1997) 7578 [gr-qc/9705070].

[51] P. F. González-Díaz, P. Martín-Moruno and A. V. Yurov, "A graceful multiversal link of particle physics to cosmology", Grav. Cosmol. 16 (2010) 205 [arXiv:0705.4347 [astro-ph]].

[52] M. Visser and S. E. C. Weinfurtner, "Vortex geometry for the equatorial slice of the Kerr black hole", Class. Quant. Grav. 22 (2005) 2493 [gr-qc/0409014].

[53] K. Rosquist, "A Moving medium simulation of Schwarzschild black hole optics", Gen. 
Rel. Grav. 36 (2004) 1977 [gr-qc/0309104].

[54] H. Stephani et al., Exact solutions of Einstein's field equations, (Cambridge University Press, 2003).

[55] J. B. Griffiths and J. Podolský, Exact spacetimes in Einstein's general relativity, (Cambridge University Press, 2009).

[56] Hawking and Ellis, The large scale structure of space-time, (Cambridge University Press, England, 1973). 